\title{
Preventing Recurrence of Severe Accidents at Nuclear Power Plants
}

\author{
-Think up Disaster Prevention along with Citizens for \\ Nuclear Safety-
}

Hosei University (Chair of the Investigation Commission on the Prevention of Severe Accidents at Nuclear Power Plants), Hiroshi Miyano Tokyo City University (Chair of the Risk Information Use Subcommittee under the Standards Committee, AESJ), Ken Muramatsu

\begin{abstract}
Nuclear power generation carries with it inherent risks associated with radioactivity. The accident that occurred at the Fukushima Daiichi Nuclear Power Plant was the manifestation of such a risk. National and local governments, scientific communities, utility companies, manufacturers, and all other stakeholders were reminded of their responsibilities with respect to their roles involving nuclear power. The accident also served as a keen reminder of the importance for them to address the essence of nuclear safety. They need to ensure safety according to their roles in the design, operation, and disaster management of nuclear power plants. Risk assessments are vital as they allow stakeholders to provide substance to the necessary safety measures, divide the requisite roles amongst themselves, and verify their effectiveness in preventing abnormal events, mitigating their impact, and preventing and mitigating any damage from an accident involving the release of radioactive substances. More extensive risk assessments are recommended to cover hitherto neglected disaster management and cleanup measures in the aftermath of an accident. Doing so is expected to make power plants considerably more resilient to accidents and ensure nuclear safety.
\end{abstract}

KEYWORDS: Nuclear safety, scientific risk, social risk, risk assessment

\section{Introduction}

The magnitude 9 earthquake that struck the Tohoku region of Japan on March 11, 2011, was one of the strongest ever recorded in the country. The Fukushima Daiichi Nuclear Power Plant was also affected by the subsequent tsunami, and the resultant damage eventually led to a nuclear accident.

The direct cause of this accident was a failure to anticipate and adequately consider natural disasters. Important contributory factors later emerged through deeper analysis. For instance,

DOI : 10.15669/fukushimainsights.Vol.3.182

(C) 2021 Atomic Energy Society of Japan. All rights reserved.

Originally published in Journal of the Atomic Energy Society of Japan (ISSN 1882-2606), Vol. 58, No. 6, p. 356-361 (2016)

in Japanese. (Japanese version accepted: February 29, 2016) 
earlier measures failed to address all of the possible types of natural disasters. Furthermore, accident management was insufficient in various respects, including an utter failure to respond to natural disasters and other external events beyond the design basis. No conceptual framework or system had been established to incorporate new scientific findings. The plant's emergency response was completely disabled by the lack of a systematic approach to the handling of the necessary equipment and the resultant failure of its vital safety functions when the supply of power as supporting components was lost. An effective emergency response could not be taken due to a failure to organize an appropriate command and decision-making system.

After the experience at the Fukushima Daiichi Nuclear Power Plant, an investigation commission was established to prevent the recurrence of severe accidents at nuclear power plants. Initially, in the fall of 2012, this commission was established following a proposal made by Hiroyuki Abe, the former president of Tohoku University. It started with his suggestion that "Every human-developed technology has been meaningfully developed. Efforts on how to use it as useful to humans are one of the important tasks of scientists and engineers." Nuclear power is no exception. Accordingly, the commission discussed the lessons that can be learned from the Fukushima Daiichi nuclear accident and the actions that need to be taken to put nuclear technologies to safe use. The conclusions of the commission were compiled into ten recommendations, which were then presented to the Nuclear Regulation Authority along with a report. These recommendations were also announced more widely to request their implementation by the relevant agencies. Most of them were put into practice after their incorporation into the new standards established by the Nuclear Regulation Authority. Unfortunately, efforts to make effective use of risk assessments remain inadequate despite this recommendation having the highest assigned priority. This is presumably due to a mixture of different reasons. One of the most important factors was probably the lack of social understanding. Hence, it was deemed necessary to explain the nature and benefits of risk assessments in order to gain the understanding of the public.

\section{Recommendations and Measures for Preventing Severe Accidents}

\section{Purpose and Background}

Anyone involved in nuclear energy must always remember the common sense belief held in other industries: there is no absolute safety. No matter how much you strive to ensure nuclear safety, the risk of an accident will always remain. Such risks must be discussed and addressed in tandem with wider society. In other words, a system must be established to promote comprehensive risk management and decision-making as a vital task that has been left to us in the wake of the Fukushima Daiichi nuclear accident. Some initiatives have already been undertaken toward this end.

In April 2013, the commission published a report on Phase I (with reference to a book published on January 20). In November 2013, the commission held a symposium aimed at enhancing nuclear power safety by adopting the risk concept, as social and scientific risks involved in nuclear. In April 2014, as a follow-up to the social and scientific risks involved in nuclear, an international symposium was held to discuss optimal countermeasures for earthquakes, tsunamis, and other natural hazards. At this symposium, a social dialogue was 
conducted in an attempt to determine how much understanding could be gained with respect to risk assessments for nuclear safety. Unfortunately, it proved quite difficult to gain an understanding of the risks and risk assessments.

In 2015, the commission sought to conduct participatory risk assessments by engaging the wider society, shifting away from the traditional approach of risk communication and efforts to seek an understanding of the risks involved. The commission began to exchange views with the municipal personnel responsible for nuclear disaster management. A workshop was also held in October to facilitate an exchange of views on risks and nuclear disaster management.

In this manner, after a process of trial and error, the commission finally began to put participatory risk management into practice.

\section{Implementing Recommendations to Address Root Causes}

The root causes of the Fukushima Daiichi nuclear accident can be categorized as follows: (1) inadequate anticipation of natural disasters; (2) insufficient accident management measures; (3) ineffective disaster management system; and (4) failure to learn lessons from international exchanges (particularly with respect to initiatives involving risk assessments). With these causes in mind, the commission concluded that they needed to re-examine whether it was appropriate to have allowed utility companies operating nuclear power plants to be responsible for ensuring safety themselves and what the national government and regulatory authorities should have done. The results were compiled into ten recommendations, which were also shared with the public. The key recommendations were as follows: ensure adequate responses to unanticipated events (Recommendation 1); implement highly evaluated world-class measures (Recommendation 2); implement concrete measures for preventing and mitigating accidents with proper recognition given to the assigned responsibilities (Recommendation 3); and engage all parties in discussions of the risks involved and the necessary countermeasures (Recommendation 4). Some of these recommendations have already been incorporated into the new regulatory standards. Many of them have been implemented with adequate equipment having been put in place.

\section{Remaining Challenges: Role of Risk Assessments}

The accidents that preceded the Fukushima Daiichi nuclear accident have inevitably involved design-related issues. Hence, the designs of nuclear power plants should be constantly revised. Many past incidents have also involved human error, other human factors, and malfunctions. Western countries were already conducting risk assessments in order to ensure safety even during unanticipated events. The Fukushima Daiichi nuclear accident revealed that Japan was lagging behind its counterparts in this respect. The new regulatory standards are mostly focused on factors involving designs, while accident management and other measures address problems associated with equipment. Other intangible soft measures remain inadequate. In contrast, Western countries have long been conducting these risk assessments in earnest to improve their measures, particularly since the Three Mile Island accident.

To comply with defence in depth, safety is pursued by implementing measures independently of the design, operation, and disaster management of nuclear power plants. The roles that these measures play are respectively assigned according to the results of risk assessments to reduce risks effectively.

The term "risk" can be expressed as the product of the probability of an event and the 
magnitude of the consequences. The same yardstick for consequences must be employed to evaluate a variety of different types of risks on an equal basis. Until now, the magnitude of consequences has been expressed as the mortality. One disadvantage of this yardstick is that it cannot adequately express smaller risks. As a possible alternative, the amount of released radioactivity can be considered as a rough indicator of environmental pollution. In Fukushima, the measures that were taken with respect to the design were insufficient, and any measures taken with respect to operations and disaster management were inadequate. As a result, almost $10 \mathrm{PBq}$ of radioactive substances ended up being released. This radioactivity did not harm people directly, but the poorly coordinated evacuation resulted in roughly 200 casualties among the sick and elderly. Appropriate preventive and mitigation measures in relation to the plant operations would have resulted in a much lower release of radioactive substances. Similarly, better preparedness in terms of disaster response would have helped to avoid the casualties caused by the poorly coordinated evacuation. A new risk target of $100 \mathrm{TBq}$, for instance, is feasible as long as appropriate measures are adopted in the relevant areas as well as with respect to the design and operations. In other words, ensuring low risk in individual areas can ensure safety and, of course, lead to an overall risk reduction and safety.

Recommendations 3 and 4 imply that risks cannot be addressed simply by adopting hardware measures to prevent accidents involving equipment. These recommendations serve as reminders that nuclear safety must also be ensured by addressing risks associated with the design, operation, and disaster management of nuclear power plants to reduce the impact that radioactive substances have in the respective areas. Rather than pursuing absolute safety through hardware measures alone, appropriate safety measures ranging from operational management to disaster management must be considered and chosen to reduce risks while engaging the wider society in the process. A consensus-driven system must be established to steer this approach in a direction that gains support and understanding. Risk assessments are the bedrock of such a system.

\section{Risk Analysis and Assessments for Disaster Management}

\section{Application of Risk Assessments for Disaster Management in General}

Society faces a variety of threats and hazards, such as earthquakes, tsunamis and volcanic eruptions as well as the heavy rains caused by typhoons. An example is presented to explain a suitable evacuation plan for addressing threats and hazards, carrying out an evacuation in response to an escalating event, and implementing any other measures for disaster management.

In the management of disasters in general, risk assessments are conducted with the aim of minimizing the total number of human casualties among residents as a risk. Figure $\mathbf{1}$ shows some examples of hazard factors, which are threats to society that can cause a disaster. In this context, starting from the occurrence of a disaster from these hazard factors, a risk is considered a combination of the likelihood of a hazard event as a disaster (a hazard map is usually produced) - and the anticipated extent of damage to residents. A disaster management plan should be developed by accurately evaluating what types of measures can change the risks levels and to what extent.

\section{General Disasters and Risk Assessments}

Choosing the right indicator to monitor the threats posed by hazards is challenging. The 
indicator must vary with time and facilitate decision-making related to the preparation and initiation of an evacuation. Decisions concerning the issuing of evacuation orders and the like must be made early enough to ensure proper sheltering and evacuation.

Once a decision has been made to initiate an evacuation, the hazards and threats involved in the evacuation process must be considered along with their likelihood and feasibility. Any disaster management planning that is conducted in advance of an evacuation decision should bear in mind that the ultimate risk levels depend on which of the given options is chosen. Obviously, disaster management does not end with the evacuation. Risk assessments may be applicable and useful in deciding how the reconstruction and the restoration of normalcy should be pursued after the evacuation.

In the event of a river flooding due to a typhoon, for example, the extent of damage will vary depending on which of the following choices is taken: wait at home, evacuate during the flooding, or request a rescue.

As shown in Figure 2, a hazard must be quantified for a suitable response to be taken. A hazard is quantified along the vertical axis, which changes over time. A decision on the waiting at home or initiation of an evacuation is made while taking into account the necessary amount of time and the threshold level of the hazard. A decision is not made according to a blanket procedure. It depends on the intended targets, their respective environments, and

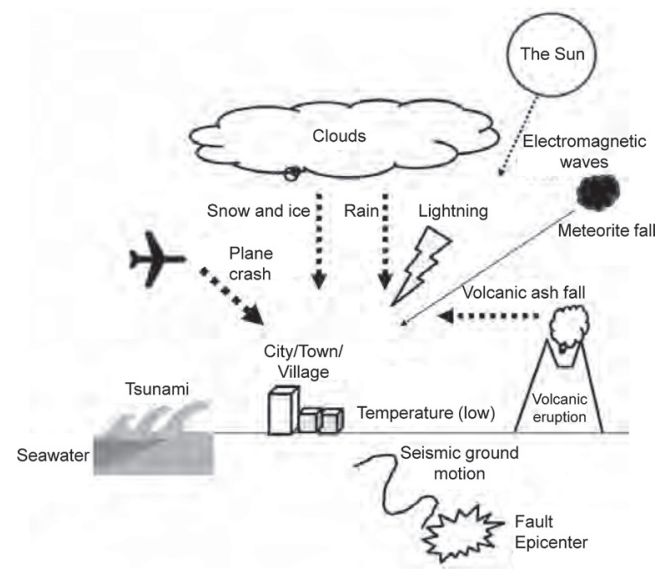

Figure 1 Examples of threats to society

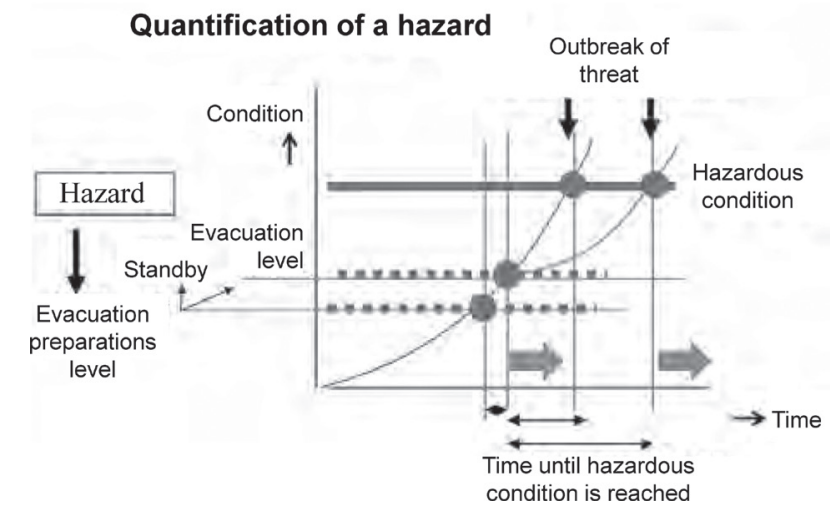

Figure 2 Decision-making related to an evacuation based on a quantified hazard 
other conditions. An evacuation may even begin during the standby phase. The analysis of hazards should also be part of risk analysis.

\section{Application of Risk Assessments for Nuclear Disaster Management}

This section describes how risk assessments are employed for nuclear disaster management in a way that is analogous with their application in the management of disasters in general. Hazards involving nuclear disaster management pose a threat to residents just like earthquakes, tsunamis, typhoons, and the like in the context of the management of disasters in general. A threat to residents involved in nuclear disaster prevention is the release of radioactive substances from nuclear power plants. Theoretically, an evacuation can be decided based on an appropriately defined indicator. In current practice, however, the evacuation of residents from a particular area is initiated as soon as radioactive substances are released from a power plant or the national government orders it. The same evaluation method is employed despite the fact that the risks involved in evacuations from different starting points vary according to the evacuation routes and conditions. In a risk assessment, appropriate risk reduction measures can be obtained by considering what is defined as a risk, what constitutes an acceptable risk, and how a risk can be reduced.

Nuclear disaster management must take into consideration the risks borne by residents from an extended area. How such risks should be aggregated as a social risk is something that will need to be considered in the future along with an effective means of applying risk values.

\section{Relationship Between the Management of Nuclear Disasters and That of Disasters in General}

The management of nuclear disasters seems no different from that of disasters in general with respect to the ways in which events escalate and how risks are assessed. Figure 3 provides an overview of risk analysis and assessments. Instead of winds, flooding, and the other hazards posed by general disasters, nuclear disaster management deals with the diffusion and fallout of radioactive substances. In sharp contrast to the visible hazards associated with general disasters, nuclear accidents require measures for dealing with the invisible hazards posed by radioactive substances. The important challenge is how such differences should be factored

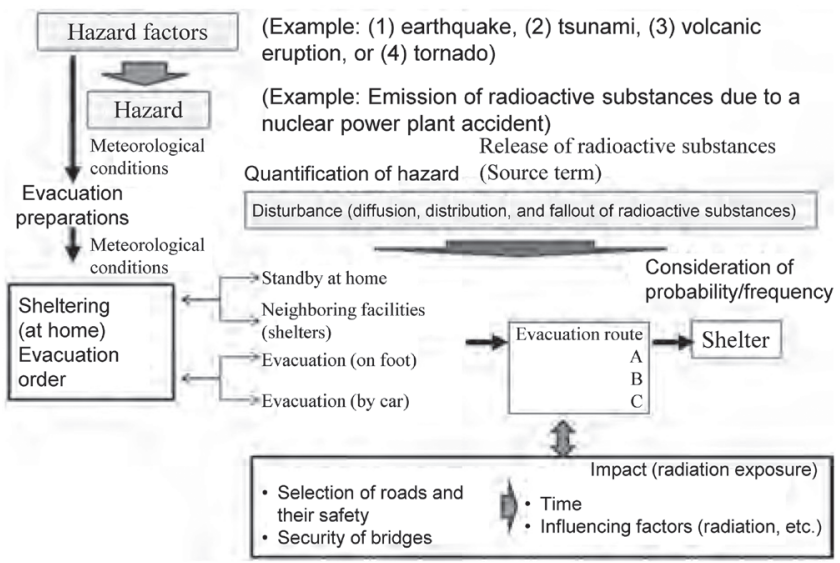

Figure 3 Hazards and risk assessments in nuclear disaster management 
into risk analysis and assessments.

The same assessment approach is taken with respect to the risks involved in the escalation of nuclear accidents inside power plants and the measures taken outside. The key here is the method by which escalating events are identified in the example shown in Figure 2. In other words, without the quantification of a hazard to identify an escalating event, a suitable response cannot be taken. At the same time, when an accident at a power plant progresses and radioactive substances are released from the power plant, if the extent of this release cannot be ascertained, the residents are considered to be in an emergency. However, it is not just difficult to understand the situation and difficult to take disaster prevention measures after an accident occurs, it is extremely difficult.

Risk assessments, therefore, must be performed while bearing in mind that the target events are invisible.

\section{Engaging the Wider Society in Risk Assessments}

Communities and nuclear power plants alike suffer in the event of a natural disaster. Such events may lead to complex nuclear emergencies, and the community must consider how to deal with compound events. Until now, the risk assessments performed at nuclear power plants have been focused on damage to the equipment, reactor cores, and primary containment vessels. Level 3 probabilistic risk assessments used to be conducted using a simple model to assess the risks to the public, and they supposedly ensured a high level of safety. Nonetheless, the risks posed by nuclear accidents must ultimately be carried by the local communities and society as a whole. Given this, simply assessing the risks associated with an escalating event from the perspective of nuclear power plants is not enough. Risk assessments must be conducted from the perspective of local residents by considering which risks should be borne, to what extent they should be borne, and how a disaster should be managed. These approaches must be combined in the pursuit of nuclear safety.

\section{Nuclear Safety with Participatory Disaster Management}

\section{Application of Risk Assessments for Disaster Management}

The resultant consequences and probability of disasters can be reduced through the appropriate design, operation, and disaster management of nuclear power plants.

Given the increasingly complex nature of the hazard factors, the authors believe that nuclear safety can be ensured by considering and addressing all of the various types of risks involved in both natural and human-induced events in a comprehensive manner. Until now, the risk assessments conducted by the nuclear sector have been focused on the safety of equipment at nuclear power plants. In terms of disaster management, though, they simply suggested the performance of basic additional assessments because they believed that requiring an evacuation would be sufficient in the event of a highly unlikely accident. However, such risk assessments were seldom conducted. Nuclear safety and disaster management should be pursued from the perspective of local residents. Risks should be defined by involving the public in the thinking process. The key task going forward is to determine how this process should be managed and who should assume responsibility for it.

Risk assessments for the use of nuclear power require definitions of the risks involved as well as clarification of how scenarios should be considered and how the assessment results 


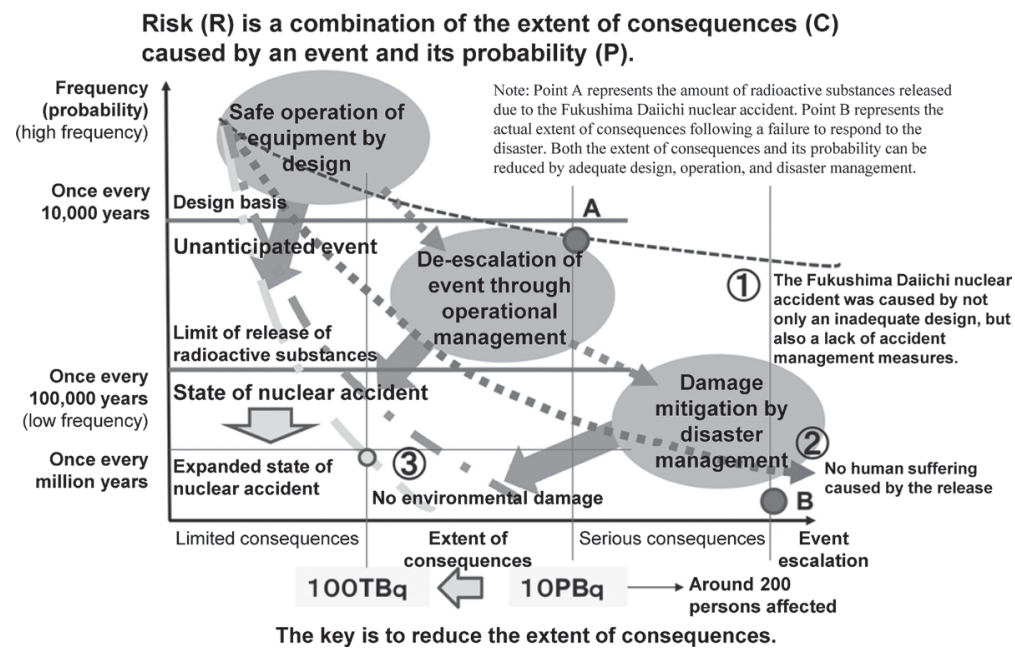

Figure 4 Total risk assessment to ensure safety

should be applied. As shown by the schematic illustration in Figure 4, such assessments are intended to reduce both the degree of consequences along the horizontal axis and the probability or likelihood along the vertical axis. Risk reduction during the design phase is pursued to ensure the safe operation of equipment by adopting a robust design, while risk reduction during the operational phase is intended to deescalate events through appropriate management and respond to events that may lead to an accident beyond the design basis. Combined with these efforts, the performance of disaster management to mitigate damage in accordance with the figure can maximize nuclear safety and minimize the risks involved in a nuclear accident. Going forward, risk management should engage all stakeholders as a whole society; in other words, local community members, the public, nuclear experts and risk experts, manufacturers, municipalities, and regulatory. The application of risk assessments in disaster management serves as an important interface toward achieving this goal. Such a practice is expected to ensure nuclear safety and reduce risks more effectively.

\section{Engaging the Wider Society in the Pursuit of Nuclear Safety}

The discussion so far has covered risk perceptions in society and risk communication as a means of dialogue. Nuclear safety must be pursued in every phase-from the design of a nuclear power plant through its siting, construction, and operation to disaster managementby implementing risk mitigation measures to prevent the potential risks of the radioactive substance release from becoming imminent threats. Otherwise, well-balanced and effective measures must be implemented to reduce the overall risks. This comprehensive approach to risk mitigation and risk assessments is unprecedented. In the past, concerns were focused on the balance between the risk assessments and risk reduction measures devised by experts on behalf of the nuclear sector. Going forward, participatory risk management must be pursued by engaging the public. Similarly, measures adopted in disaster management to mitigate risks ought to be considered by adopting the concept of risks. Measures aimed at ensuring nuclear safety will hopefully be established by the wider society in a more transparent manner so that the public can keep track of them. 


\section{Conclusions: Why Are Risk Assessments Important?}

This commentary has discussed how social acceptance of risk assessments can be gained by promoting a deeper understanding of their importance. In fact, even in the nuclear sector, many people and groups still do not understand the importance of risk assessments.

Why are risk assessments important?

One possible reason for their importance is the need to minimize phenomena that cannot be anticipated. Many scenarios can be adopted to reduce unknown factors and minimize phenomena that cannot be anticipated. Important decisions can be made objectively by adopting common judgement criteria and quantified risk values in risk assessments of matters ranging from the design and operation of nuclear power plants to disaster management. Doing this enables suitable safety measures to be devised. Disaster management combined with the concept of risks can help engage the wider society in the consideration of matters such as the risk assessment results, the safety goals to be assigned, associated uncertainty, and unknown factors. Nuclear Scientists, engineers, and the public can share the same perspective on the decisions that are made. It would be beneficial to engage the wider society in discussions of what the risks are and how they can be mitigated through joint action.

Risk assessments have already been conducted with respect to the risk factors associated with nuclear power plants. Initiatives aimed at applying risk assessments to disaster management as explained in this commentary will help to formulate a consistent practice of conducting risk assessments in every phase, from manufacturing and operations all the way through to disaster management, and thereby stimulate cross-sectional discussions and partnerships. To this end, it would be necessary to develop human resources in the conducting of risk assessments and promote risk literacy among people. (February 26, 2016)

\section{Acknowledgments}

This study was conducted as part of the activities undertaken by the Japan Association of Technology Executives with funding from the Watanabe Memorial Foundation for the Advancement of Technology and assistance from the Japan Science and Technology Exchange Center.

\section{General References}

1) Work Compiled by the Investigation Commission for Preventing Severe Accidents at Nuclear Power Plants, Recommendations for Nuclear Policy (Part 1): Key Measures That the Japanese Government and Nuclear Sector Need to Implement to Prevent the Recurrence of a Severe Accident at a Nuclear Power Plant [in Japanese], Japan Science and Technology Exchange Center, January 2016.

2) Hiroshi Miyano: 12th Scientific Lecture Meeting, Scientific Risks and Social Risks [in Japanese], 2B-3-1, P357-359, Japan Society of Maintenology, 2015.

3) Hiroshi Miyano: Rethinking Nuclear Power in Pursuit of Nuclear Safety [in Japanese], Energy Review, March 2016. 